\title{
Role of Explicitly Included Solvents on Ultrafast Electron Injection and Recombination Dynamics at $\mathrm{TiO}_{2} /$ Dye Interfaces
}

Wei Ma ${ }^{1,2}$ ‡, Yang Jiao ${ }^{2}$ ‡, Haibo Li ${ }^{1}$, Haizhong Guo ${ }^{4}$, Efthimios Kaxiras ${ }^{3 *}$, Sheng $\operatorname{Meng}^{2,5 *}$

${ }^{1}$ Ningxia Key Laboratory of Photovoltaic Materials, Ningxia University, Yinchuan, Ningxia 750021, P.R. China

${ }^{2}$ Beijing National Laboratory for Condensed Matter Physics, and Institute of Physics, Chinese Academy of Sciences, Beijing, 100190, P. R. China

${ }^{3}$ Department of Physics and School of Engineering and Applied Sciences, Harvard University, Cambridge, Massachusetts 02138, USA

${ }^{4}$ School of Physics and Engineering, Zhengzhou University, Zhengzhou, Henan 450001, P. R. China

${ }^{5}$ Collaborative Innovation Center of Quantum Matter, Beijing, P. R. China 


$$
t=1.10 p s
$$

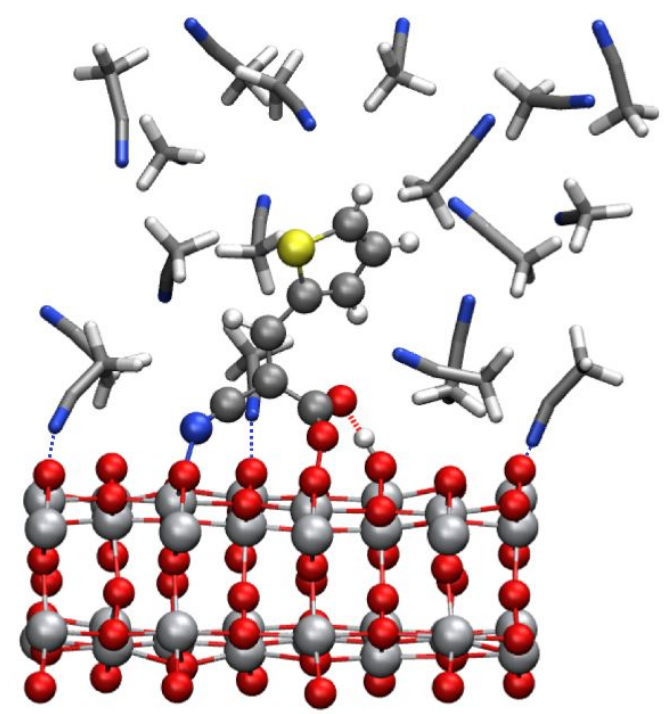

Figure S1. Snapshot of interface chemical structure at $t=1.10 \mathrm{ps}$ in AIMD simulations of solvent system. A maximum of three acetonitrile molecules are observed to bind simultaneously through their nitrogen atoms to surface low-coordination $\mathrm{Ti}(\mathrm{IV})$ centers, out of the six available sites of the $\mathrm{TiO}_{2}$ semiconductor substrate, while two additional sites are occupied by the dye molecule. 

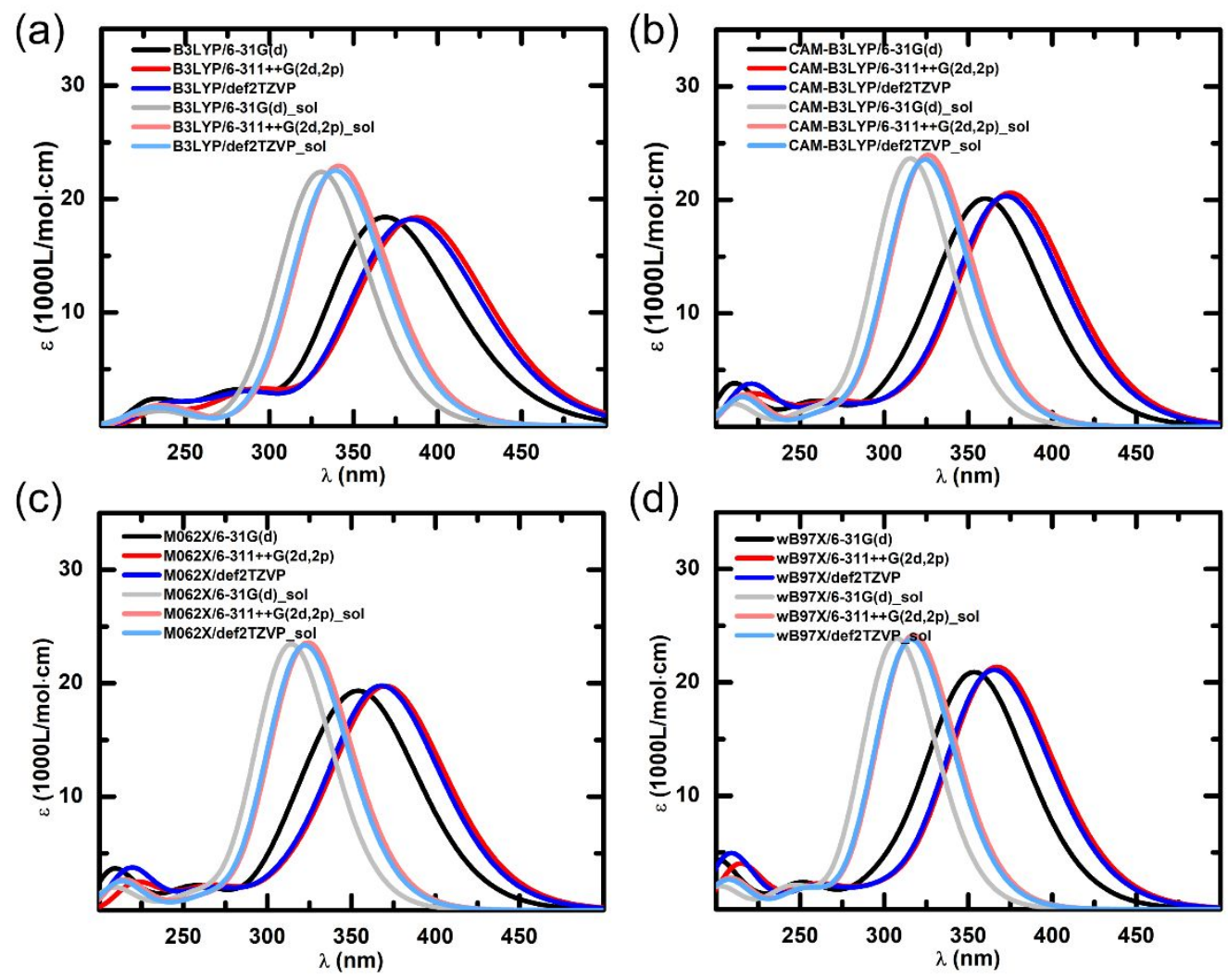

Figure S2. Absorption spectra of M0 dye molecule at T $=350 \mathrm{~K}$ for the fully solvated system and the vacuum system from TDDFT calculations using (a) B3LYP, (b) CAM-B3LYP, (c)M06-2X and (d) wB97X functionals with different basis sets. The dark lines denote the light-absorption of M0 molecule in vacuum, while the light lines show the absorption spectra of M0 molecule in solvated system. 


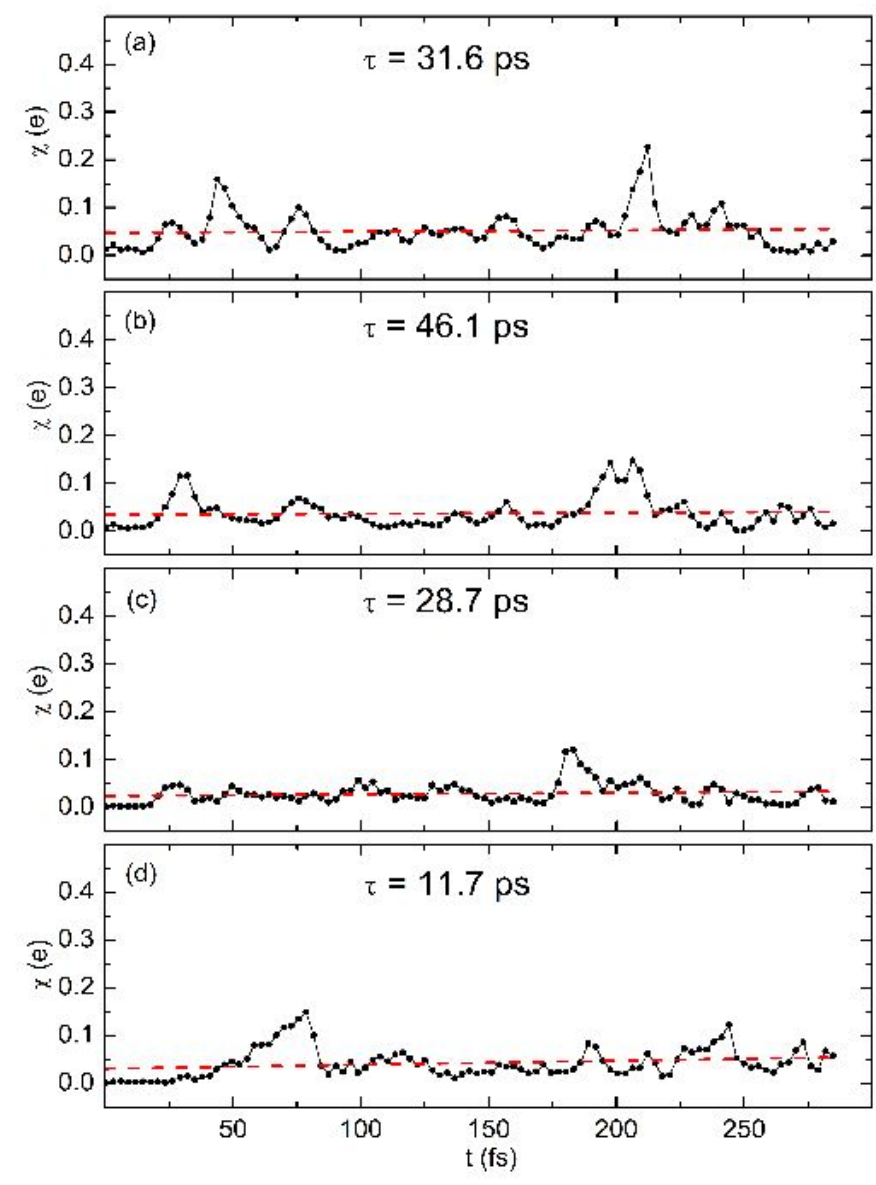

Figure S3. Photo-excited electron distribution on the M0 molecule after injected into the $\mathrm{TiO}_{2} \mathrm{CB}$ at the organic dye/ $\mathrm{TiO}_{2}$ heterointerface. Four trajectories correspond to excitations from $\mathrm{HOMO}$ of the dye to different energy states of $\mathrm{TiO}_{2} \mathrm{CB}(\mathrm{H} \rightarrow \mathrm{L}+1$, $\mathrm{H} \rightarrow \mathrm{L}+2, \mathrm{H} \rightarrow \mathrm{L}+3, \mathrm{H} \rightarrow \mathrm{L}+4)$. Dashed lines are linear fittings of the four trajectories. 